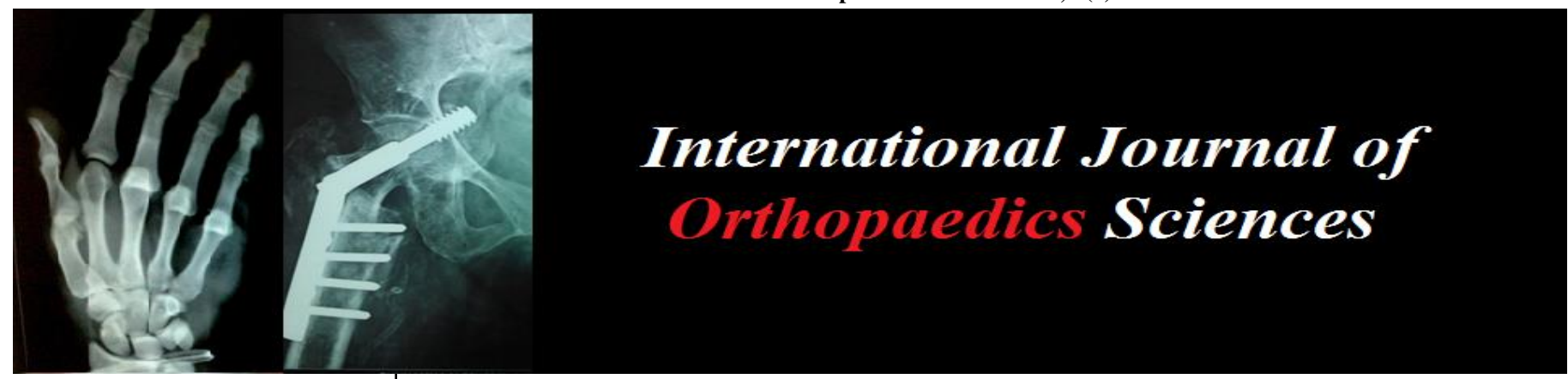

E-ISSN: 2395-1958

P-ISSN: 2706-6630

IJOS 2021; 7(3): 727-732

(C) 2021 IJOS

www.orthopaper.com

Received: 04-05-2021

Accepted: 06-06-2021

Dr. Kandarpkumar Kanubhai

Patel

Senior Resident, Department of Orthopedics, Government

Medical College and New Civil

Hospital, Surat, Gujarat, India

Dr. Hari J Menon

Professor and Head, Department

of Orthopedics, Government

Medical College and New Civil

Hospital, Surat, Gujarat, India

Dr Viren Atulbhai Umrethiya Junior Resident, Department of Orthopedics, Government Medical College and New Civil Hospital, Surat, Gujarat, India
Corresponding Author:

Dr. Kandarpkumar Kanubhai

Patel

Senior Resident, Department of Orthopedics, Government

Medical College and New Civil

Hospital Surat, Gujarat, India

\section{A study of clinical outcomes of only decompression versus decompression with instrumented fusion for surgical management of lumbar canal stenosis in tertiary health care center in south Gujarat}

\author{
Dr. Kandarpkumar Kanubhai Patel, Dr. Hari J Menon and Dr. Viren \\ Atulbhai Umrethiya
}

DOI: https://doi.org/10.22271/ortho.2021.v7.i3k.2824

\begin{abstract}
Lumbar spinal stenosis is one of the most common conditions seen in elderly. It is a degenerative progressive disorder which leads to considerable disability. Patients not responding to conservative management requires surgical intervention. Different modalities for surgical interventions available. Decompression by laminectomy discectomy or micro lumbar discectomy or micro endoscopic discectomy can be done. Decompression with addition of instrumented fusion with help of pedicle screws and rods with or without TLIF/PLIF. Hence to compare the clinical outcomes of decompression and decompression with instrumented fusion this study is done. In most patients, conservative management is considered for relieving pain and functionally improving walking and standing time. The patients not improving with conservative management for at least 6 weeks and having clinically and radiologically lumbar canal stenosis requires surgical intervention.

Apart from Minimal invasive surgery Decompression with or without fusion has been traditional definitive therapy offered. In this study we found that surgical treatment was influenced by presenting complaint of the patient. Patients with predominant back pain were more often operated with decompression with fusion whereas patients having radiculopathy or claudication were more often operated with decompression only. In this study clinical outcomes of decompression only and decompression with fusion were found similar. However, blood loss during surgery, duration of surgery and duration for return to work/occupation were more in decompression with fusion surgery which was found statistically significant.
\end{abstract}

Keywords: stenosis, decompression, instrumented fusion, ODI, VAS

\section{Introduction}

Lumbar canal stenosis is a progressive degenerative disorder involving spinal motion segment. Instability and hypermobility of the facet joints leads to facetal hypertrophy which causes degeneration of intervertebral disc. Calcification and hypertrophy of ligamentum flavum are also contributing factors. Anatomically reduced spinal canal diameter is responsible for neurogenic claudication and radicular pain.

Surgical treatment for management of Lumbar canal stenosis consists of laminectomy discectomy. Although decompression with instrumented fusion has also been used as a surgical management in cases of LCS. Meta-analysis and systemic reviews have not shown clear evidence regarding superiority in terms of clinical outcomes from these surgeries. So, this study aims to evaluate the outcomes of these surgeries and compare their outcomes.

\section{Materials and Methods}

After getting approval from ethics committee this prospective observational study was conducted. The study group included 30 patients. 15 patients in each category of surgery 1) Decompression and 2) Decompression with instrumented fusion. All patients presenting to New civil hospital, Surat with low back pain with / without radiculopathy satisfying the inclusion criteria as mentioned below are included in this study. The study was conducted between 2018 to 2019 . 


\section{Inclusion criteria}

1. Patients registered in NCH, Surat during period of 20182019

2. Patients having Lumbar canal stenosis diagnosed clinically and radiologically, not improving on conservative care for at least 6 weeks

3. Patients operated with a decompression procedure with/without instrumented fusion through posterior approach

\section{Exclusion Criterias}

1. Previous history of spine surgery

2. Decompression / instrumentation through an anterior approach

3. Tandem stenosis

4. Lumbar instability motion $>4 \mathrm{~mm}$ on dynamic lumbar spine X-rays

All the patients included in this study underwent a thorough clinical and neurological examination. They also underwent plain X-ray of lumbosacral spine AP and lateral (dynamic views) and MRI of the lumbosacral spine. Oswestry disability index and Visual Analogue score were used to evaluate clinical condition of the patient. Routine investigations were done. Patients failed to improve on conservative care for at least 6 weeks were counseled for surgery, merits and limitations.

All patients were operated on simple table on bolsters in prone position under inhalational hypotensive general anesthesia via posterior midline approach.

For decompression - laminectomy, laminotomy or fenestration performed For decompression with fusion - laminectomy along with posterior stabilization with help of pedicle screws and rods with / without transforaminal lumbar interbody fusion (TLIF)/ posterior lumbar interbody fusion (PLIF)/ posterolateral fusion (PLF). Intraoperative findings were documented regarding extent of decompression, integrity of facets and bleeding. Intraoperatively inj. Tranexamic acid and local hemostatic were used to reduce blood loss.

Postoperatively patients were rehabilitated in stages with lumbosacral brace. Sutures were removed on $11^{\text {th }}$ post of day. Patients were mobilized as soon as pain permitted averagely on $3^{\text {rd }}$ post op day and spinal exercises were started after $3^{\text {rd }}$ post op week in form of back extension exercises and supported sitting. Post-operative clinical evaluation was done with help of Oswestry disability index and Visual analogue scale.

\section{Results}

A total of 30 patients were included in the study group. 15 patients were operated with decompression and 15 patients were operated with decompression with instrumented fusion. The mean age of the study group was 49.4 years (Range 2270 years). Peak incidence was in $6^{\text {th }}$ decade followed by $5^{\text {th }}$ decade. Among 30 patients 19 were male and 11 were female. Among 19 male patients 8 were operated with decompression with instrumented fusion and among 11 female patients 7 were operated with decompression with instrumented fusion. Rest were operated with decompression only.

Patients having presenting complaint of predominant back pain were more often treated with decompression with fusion whereas patients having radiculopathy or claudication were treated with decompression only.

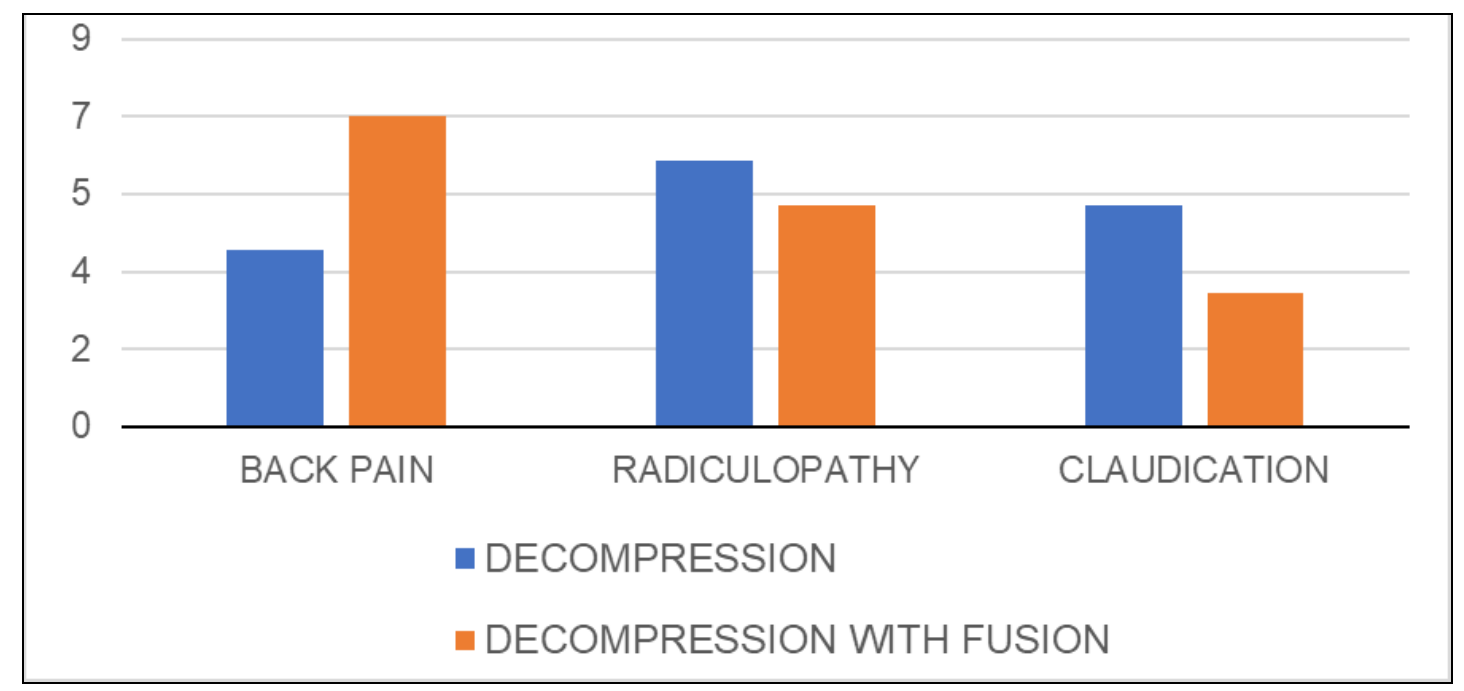

Graph 1: Presenting complain and management

Average blood loss for decompression was $144 \mathrm{ml}$ and decompression with fusion was $316 \mathrm{ml}$. which was statistically significant. (According to independent unpaired $t$ test $\mathrm{t}$ value of this test is 86.53. but critical $\mathrm{t}$ value for confidence level $95 \%$ and degree of freedom 29 is 2.045) Average duration for surgery for decompression was around 2 hours and for decompression with fusion was around 3 hours 15 minutes. According to independent unpaired t test $t$ value of this test is 2.09 , but critical $t$ value for confidence level 95 $\%$ and degree of freedom 29 is 2.045. So there was statistically significant difference in duration of both types of surgeries.

Table 1: ODI score preop and postop

\begin{tabular}{|c|c|c|c|}
\hline & All Cases & Decompression & Decompression With union \\
\hline PREOP & 48.33 & 44.13 & 52.53 \\
\hline Post Op 1 Month & 29.06 & 23.87 & 34.26 \\
\hline Post Op 3 Months & 19.37 & 16.54 & 22 \\
\hline Post Op 6 Months & 12.44 & 10.77 & 14 \\
\hline
\end{tabular}


Patients who were operated with Decompression with fusion had higher ODI preoperatively as compared to Decompression only group. In both groups' patients have maximum improvement in $1^{\text {st }}$ post op month with gradual further improvement in sequential follow ups? Both groups have similar results in terms of improvement in ODI scores in 6 months follow up. 2 patients (1 from each group) have increased ODI score post operatively. Both patients were treated conservatively with oral analgesics till last follow up (6 months).

Table 2: Improvement in ODI score compared to Preop ODI in Postop follow up

\begin{tabular}{|c|c|c|c|}
\hline Follow Up & All Cases & Decompression & Decompression + Fusion \\
\hline 1 Month & $19.27(39.87 \%)$ & $20.26(45.90 \%)$ & $18.27(34.78 \%)$ \\
\hline 3 Months & $28.96(+20.07)$ & $27.59(+16.62 \%)$ & $30.53(+23.34 \%)$ \\
\hline 6 Months & $35.89(+15.27)$ & $33.36(+13.07 \%)$ & $38.53(+15.22 \%)$ \\
\hline
\end{tabular}

According to independent unpaired $t$ test $t$ value of this test is 0.081. But critical $\mathrm{t}$ value for confidence level $95 \%$ and degree of freedom 29 is 2.045 . So, there is no significant difference in clinical outcomes of decompression and decompression with instrumented fusion.

Table 3: Visual analog scale pre-op and post-op

\begin{tabular}{|c|c|c|c|}
\hline & All Cases & Decompression & Decompression + Fusion \\
\hline Preop & 6.16 & 5.8 & 6.53 \\
\hline Post Op 1 Month & 2.3 & 2.06 & 2.53 \\
\hline Post Op 3 Months & 1.37 & 1.23 & 1.5 \\
\hline Post Op 6 Months & 1.19 & 1.15 & 1.21 \\
\hline
\end{tabular}

Table 4: Improvement in Visual analog scale

\begin{tabular}{|c|c|c|c|}
\hline Follow Up & All Cases & Decompression & Decompression + Fusion \\
\hline 1 Month & $3.86(62.66 \%)$ & $3.74(64.48 \%)$ & $4(61.25 \%)$ \\
\hline 3 Months & $4.79(+15.10 \%)$ & $4.57(+14.31 \%)$ & $5.03(+15.77 \%)$ \\
\hline 6 Months & $4.97(+2.92 \%)$ & $4.65(+1.38 \%)$ & $5.32(+4.45 \%)$ \\
\hline
\end{tabular}

According to independent unpaired t test $t$ value of this test is 1.14. But critical t value for confidence level $95 \%$ and degree of freedom 29 is 2.045 . So, there is no significant difference in clinical outcomes of decompression and decompression with instrumented fusion.

Average duration for returning to the occupation was around
9 weeks in decompression group and 10 weeks in decompression with fusion group in decompression with fusion group. It was clinically significant. (According to independent unpaired $t$ test $t$ value of this test is 10.71 . but critical $\mathrm{t}$ value for confidence level $95 \%$ and degree of freedom 29 is 2.045.)

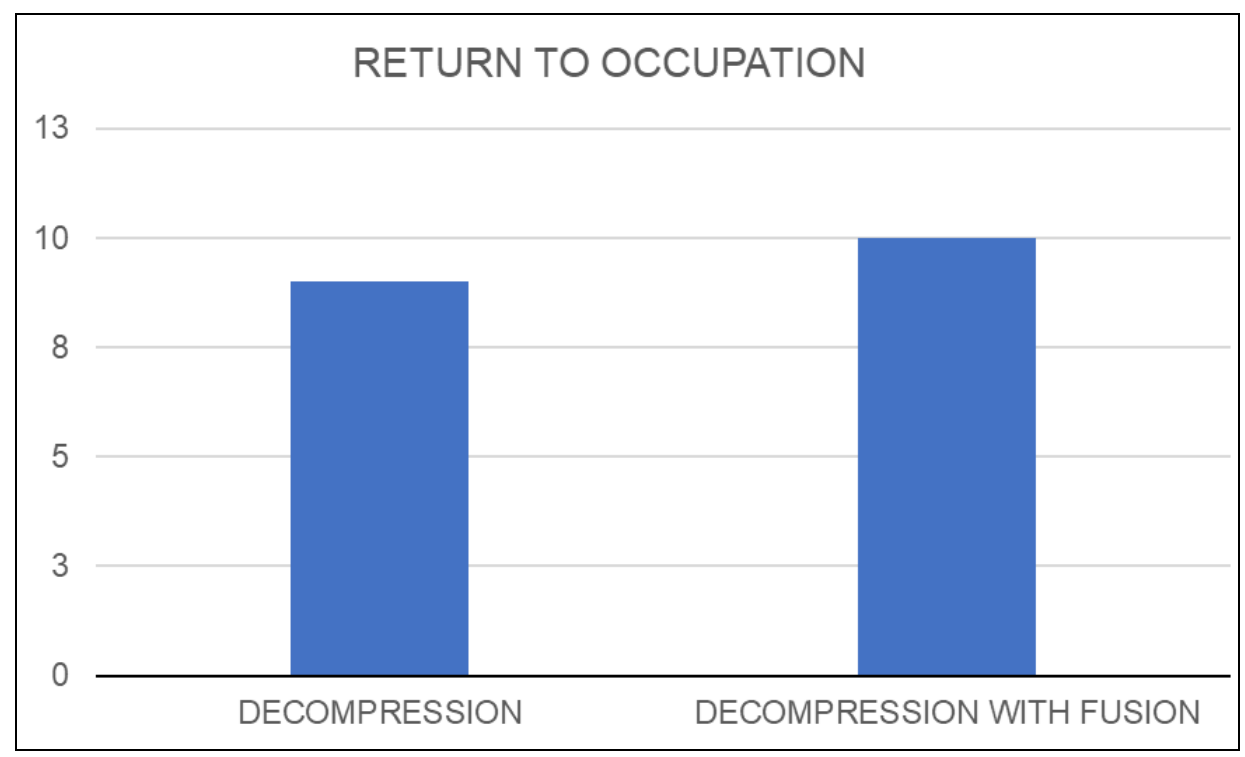

Graph 2: Return to occupation (Duration in weeks)

One patient from decompression with fusion group had Dural tear which was treated with watertight subcutaneous closure, air tight skin closure and healed uneventfully. One patient from decompression with fusion group had post-operative left sided Ankle dorsiflexion and EHL weakness (power 3/5). He was treated conservatively. In $1^{\text {st }}$ post op month patient weakness improved to power $4 / 5$ and in final follow up at 6 months patient had power $5 / 5$. One patient from each group had infection postoperatively and had serous discharge from stich line. Both patients were treated with oral antibiotics according to culture and sensitivity reports and stich line healed by $3^{\text {rd }}$ post op week.

\section{Discussion}

In our study mean age group for Lumbar canal stenosis surgery was 49.4 years and having male preponderance 19 
(63\%) patients as compared to 11 (37\%) female patients. Study conducted in 2015 by Chunyue Duan ${ }^{[1]}$, they found mean age for decompression only was about 71.1 years and 68.3 years in fusion group. No significant intergroup difference was found with respect to age, sex ratio or surgical levels.

In this study we found that patients operated with decompression only had lower ODI and VAS score as compared to Decompression with fusion group. However, both groups had similar results in terms of improvement in clinical outcomes. Duration for surgery for decompression with fusion is longer as compared to Decompression only. Similarly, blood loss is also more in Decompression with fusion group. These findings were statistically significant. In our study patients with predominantly back pain were more often operated with decompression with fusion whereas radiculopathy and claudication were operated with decompression only more often.

In this study we found that clinical outcomes in forms of improvement in ODI and VAS of decompression and decompression with fusion has similar results. Li et al. concluded that Decompression + Fusion showed less favorable outcomes in terms of ODI, length of hospital stays, and blood loss in comparison with Decompression alone [2]. They did not find any significant difference in VAS and major device-related complications, which is similar to our findings. Pasquale Donnarumma et al in 2016 suggested that posterior decompression as the preferred option in his retrospective study ${ }^{[3]}$. Sometimes the choice of the surgeon is to stabilize the spine even in the absence of micro-instability.

Another international study showed that both decompression and decompression with fusion groups resulted in reduced VAS. Aihara et al. reported higher postoperative scores in the D alone group ${ }^{[4]}$. However, their results were not statistically significant. Also, less blood loss and shorter post-operative hospitalization were observed in the Decompression alone group.

Forsth et al. randomized 68 patients to decompression alone and 67 to decompression and fusion ${ }^{[5]}$. No statistical or clinical differences were found between the groups at 2-year follow-up. Ghogawala et al. compared laminectomy alone with laminectomy plus instrumented fusion (66 patients included) at 2-, 3- and 4-year follow-up ${ }^{[6]}$. They found the physical health-related quality of life to be slightly better and more clinically meaningful in the fusion group.

Several registry studies have been published regarding clinical outcomes of surgeries for lumbar canal stenosis over the past few years. A study of 213 patients (56 operated on with decompression alone) from one center participating in the Spine Tango Registry showed better patient-reported outcomes when instrumented fusion was added to decompression. In that study, $86 \%$ of the fusion group versus $70 \%$ of the decompression alone group $(p=0.01)$ were defined as having a good outcome. When controlling for gender, age, comorbidity, number of levels operated on and baseline outcome scores in a multivariate regression analysis, fusion was a significant unique predictor for a 'good' global outcome.

In our study, patients operated with decompression only had better results in immediate post op period and returned to their occupation/routine daily activities early as compared to fusion group. It was statistically significant. In another recently published study from Swespine, 839 patients (245 with decompression alone) were analyzed in relation to preoperative pain patterns. At 1-year follow-up, after adjusting for age, gender, duration of symptoms, earlier surgery and smoking, the fused patients with predominant back pain had significantly greater reduction in pain and ODI than the non-fused patients. At the 2-year follow-up, no differences were found regardless of pain pattern.

Grob et al. ${ }^{[7]}$ Also reported no differences between decompression or decompression and fusion in outcomes at a mean follow up of 28 months. Mannion ${ }^{[8]}$ examined the outcomes of lumbar decompression surgery without fusion, found pain and disability showed minimal change in the 5year period after surgery. Niggemeyer et al. ${ }^{[9]}$ reported the similar findings in a meta-analysis by including 1668 patients with a mean follow-up of 4.7 years.

Phillips ${ }^{[10]}$ reported that the increasing use of instrumentation was correlated with the raising of mortality, the risk of complication and reoperation. Pellise ${ }^{[11]}$ showed that fixation could only improve the daily activities of the patients, but could not help to get better clinical outcomes. In addition to this, the debate about the cost-effective also still exist.

Iguchi ${ }^{[12]}$ suggest the effect of spinal instability on the outcome following decompression is less favorable with a post-operative slip. Furthermore, the addition of fusion can lead to an increased risk of life-threatening complications and a higher mortality rate, especially for the elderly patients with a high incidence of comorbidities. In elderly patients, pedicle screw fixation and interbody fusion could also have significant adverse events, cause instrument-related complications, such as postoperative complications, instrument failure, and adjacent segment degeneration (ASD). In patients with osteoporosis, transpedicular screw fixation or interbody fusion can also result in subsidence, screw failure, or nonunion. Son et al. ${ }^{[13]}$ reported that decompressive surgery alone produces good results in spinal stenosis, Ragab also showed that old age with decompressive surgery alone in lumbar spinal stenosis could get good results and does not increase morbidity associated. For older patients, decompressive laminectomy alone could minimize tissue injuries, shorten operation times, reduce perioperative morbidity, and prevent instrument-related complications.

Different surgical technique, such as decompression alone or fusion, has their own unique advantages and disadvantages. Knaub suggested that ${ }^{[14]}$ relative indications for the use of spinal instrumentation in the setting of spinal stenosis include correction of deformity, recurrent spinal stenosis with instability, degenerative spondylolisthesis, adjacent segment stenosis with instability, and multiple level fusions.

Limitations of the Study includes small sample size and duration of follow up. This study includes 30 cases (15 from each group). As sample size is small, observations obtained from this study cannot be consider same as of larger population. Follow up of this study is up to 6 months. Long period (at least 4-5 years) follow up is required to measure clinical outcomes of LCS surgeries. In this study radiological parameters were not included for outcomes of both surgeries. Addition of radiological parameters can help in measuring radiological outcomes of these surgeries, which can help us to get better evidence regarding comparison between decompression only and decompression with fusion groups.

\section{Conclusion}

Lumbar spinal stenosis results from a narrowing of the spinal canal leading to compression of nerve roots contained in the dural sac and/or foramina. In most patients, conservative management is considered for relieving pain and functionally improving walking and standing time. The patients not 
improving with conservative management for at least 6 weeks and having clinically and radiologically. Lumbar canal stenosis requires surgical intervention. Apart from Minimal invasive surgery Decompression with or without fusion has been traditional definitive therapy offered.

In this study we found that surgical treatment was influenced by presenting complaint of the patient. Patients with predominant back pain were more often operated with decompression with fusion whereas patients having radiculopathy or claudication were more often operated with decompression only. In this study clinical outcomes of decompression only and decompression with fusion were found similar.

However, blood loss during surgery, duration of surgery and duration for return to work/occupation were more in decompression with fusion surgery which was found statistically significant.
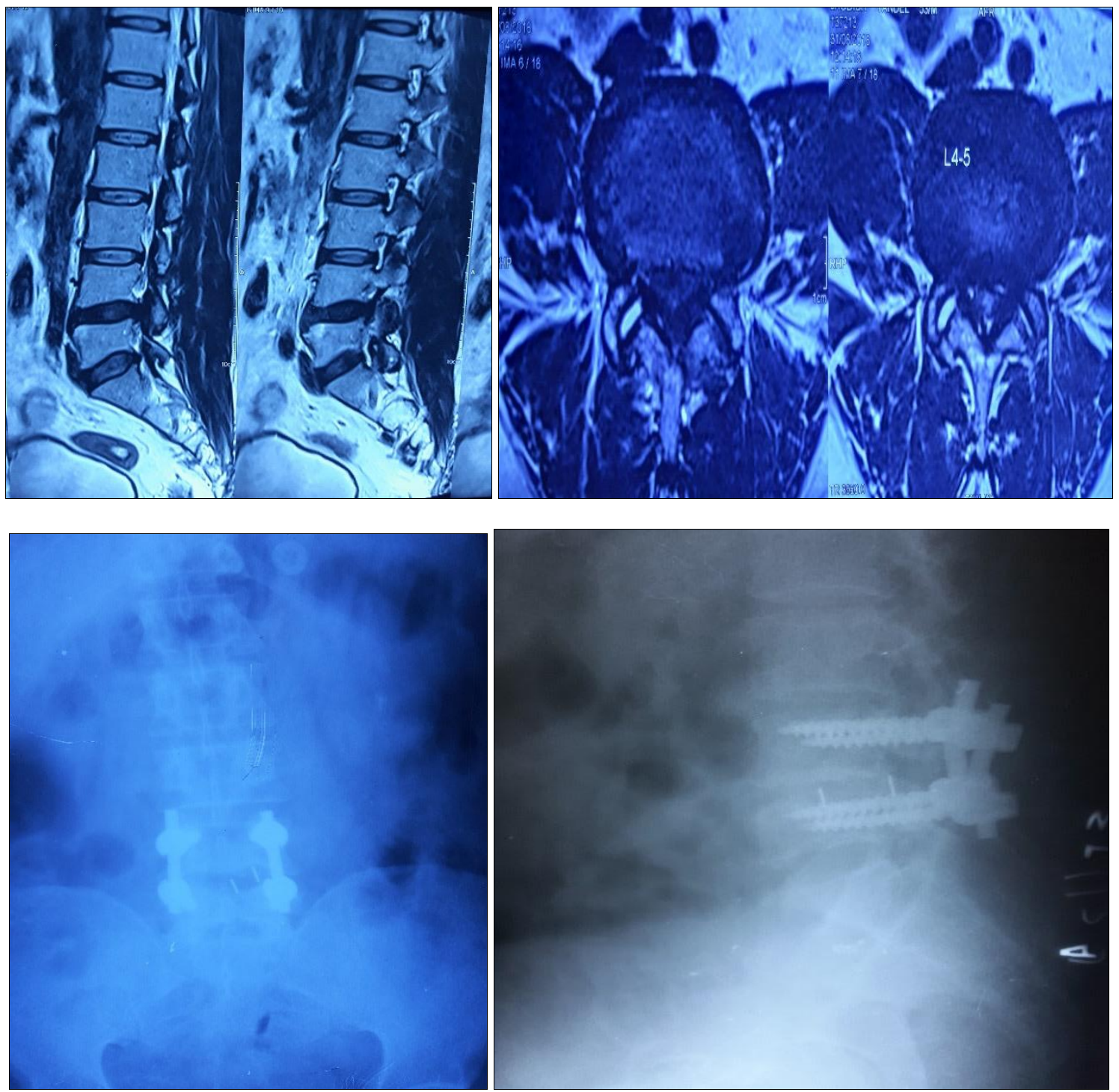

Case 1: 53 years old male patient having c/o low back pain with left lower limb radiculopathy for past 6 months. Claudication distance 500 meters. Left EHL weakness (power 3/5). On MRI LCS at L4L5 level (canal diameter $4 \mathrm{~mm}$ ). Preop ODI score 60 and VAS score 7. Operated with Decompression with fusion. Post op ODI were 32 (1 month), 24 (3 months) and 12 (6 months). Post op VAS were 3(1 month), 2(3 months) and 1 (6 months).

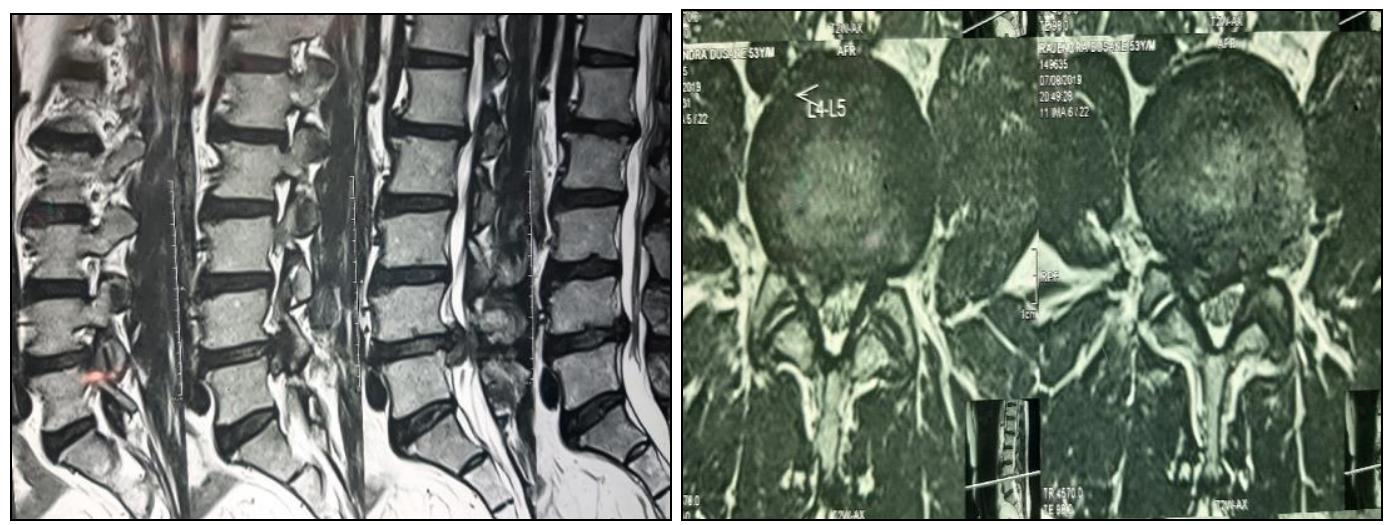



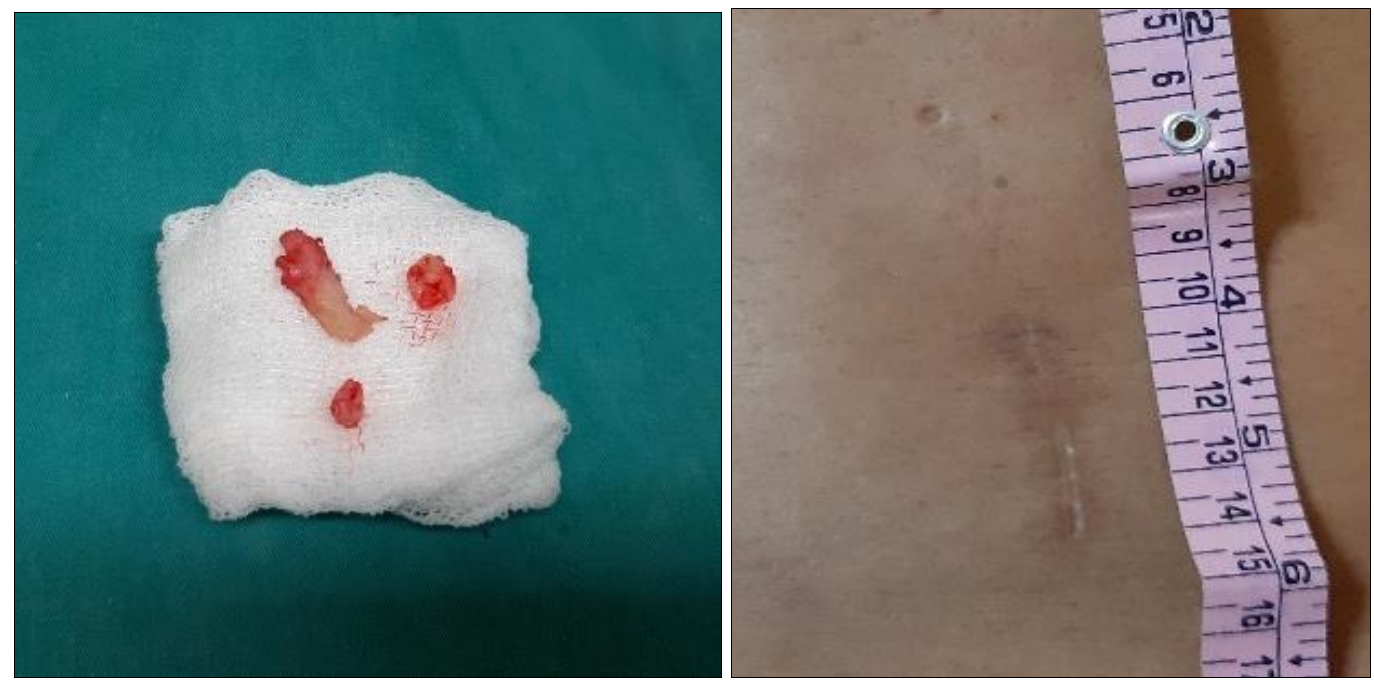

Case 2: 53 years old male patient having c/o low back pain with Right lower limb radiculopathy for 3 months. Claudication distance $1 \mathrm{~km}$. MRI - L4L5 PIVD. ODI score 25\% and VAS 6. Operated with Decompression only. Post op ODI - 10 and VAS- 2.

\section{References}

1. Comparison of decompression only versus posterior lumbar interbody fusion for lumbar nerve root canal stenosis in elderly patients Chunyue Duan, Jianzhong $\mathrm{Hu}$, Yong Cao, Xiyang Wang, Jianhuang Wu Int J Clin Exp Med 2016;9(8):16491-16497 www.ijcem.com /ISSN:1940-5901/IJCEM0028754

2. $\mathrm{Li} \mathrm{AM}, \mathrm{Li} \mathrm{X}$, Yang Z. Decompression and coflex interlaminar stabilisation compared with conventional surgical procedures for lumbar spinal stenosis: a systematic review and metaanalysis. Int $\mathrm{J}$ Surg 2017;40:60-67. 10.1016/j.ijsu.2017.02.056

3. Donnarumma P, Tarantino R, Nigro L, Rullo M, Messina $\mathrm{D}$, Diacinti D et al. Decompression versus decompression and fusion for degenerative lumbar stenosis: analysis of the factors influencing the outcome of back pain and disability. J Spine Surg 2016;2(1):52-8. doi: 10.21037/jss.2016.03.07. PMID: 27683696; PMCID: PMC5039834.

4. Aihara T, Toyone T, Aoki Y, Ozawa T, Inoue G, Hatakeyama K, Ouchi J. Surgical management of degenerative lumbar spondylolisthesis: a comparative study of outcomes following decompression with fusion and microendoscopic decompression. J Musculoskeletal Research 2012;15:1250020. 10.1142/S0218957712500200

5. Forsth P, Michaelsson K, Sanden B. Does fusion improve the outcome after decompressive surgery for lumbar spinal stenosis?: a two-year follow-up study involving 5390 patients. Bone Joint J 2013;95-B:960-965.

6. Ghogawala Z, Dziura J, Butler WE, Dai F, Terrin N, Magge SN et al. Laminectomy plus Fusion versus Laminectomy Alone for Lumbar Spondylolisthesis. N Engl J Med 2016;374(15):1424-34. doi: 10.1056/NEJMoa1508788. PMID: 27074067.

7. Grob D, Humke T, Dvorak J. Degenerative lumbar spinal stenosis. Decompression with and without arthrodesis. J Bone Joint Surg Am 1995;77:1036-1041.

8. Mannion AF, Denzler R, Dvorak J, Grob D. Fiveyear outcome of surgical decompression of the lumbar spine without fusion. Eur Spine J 2010;19:1883-1891.

9. Niggemeyer O, Strauss JM, Schulitz KP. Comparison of surgical procedures for degenerative lumbar spinal stenosis: a meta-analysis of the literature from 1975 to 1995. Eur Spine J 1997;6:423-429.
10. Phillips FM. The argument for noninstrumented posterolateral fusion for patients with spinal stenosis and degenerative spondylolisthesis. Spine 2004;29:170-172.

11. Pellise F, Hernandez A, Vidal X, Minguell J, Martinez C, Villanueva C. Radiologic assessment of all unfused lumbar segments 7.5 years after instrumented posterior spinal fusion. Spine 2007;32:574-579.

12. Iguchi T, Kurihara A, Nakayama J. Minimum 10-year outcome of decompressive laminectomy for degenerative lumbar spinal stenosis. Spine 2000;25:1754-9.

13. Son S, Kim WK, Lee SG, Park CW, Lee K. A comparison of the clinical outcomes of decompression alone and fusion in elderly patients with two-level or more lumbar spinal stenosis. J Korean Neurosurg Soc 2013;53:19-25.

14. Knaub MA, Won DS, McGuire R, Herkowitz HN. Lumbar spinal stenosis: indications for arthrodesis and spinal instrumentation. Instr Course Lect 2005;54:313319. 